\title{
Computer controlled interactive video simulation for motor skills research
}

\author{
GEOFFREY PAULL and IAN CASE \\ Curtin University of Technology, Perth, Western Australia \\ and \\ ROBERT GROVE \\ University of Western Australia, Nedlands, Western Australia
}

\begin{abstract}
Researchers examining skilled performance in the laboratory must establish contextual relevance to the usual performance environment to ensure that the obtained data truly reflect expertise in the skill under investigation. Interactive video simulation is a viable method for providing such ecological validity. This paper describes the development to date of such a simulator and outlines two sportsperformance studies which demonstrate the validity and reliability of the configuration in competitive situations. Further development of the simulator is possible through new technologies and through the assembly of specific interfaces for other motor skills. The paradigm also has potential as a training resource for teaching the specific knowledge necessary for expert levels of performance.
\end{abstract}

The high-level motor abilities of elite performers can be understood in part in terms of the extensive knowledge that they have of the environment in which they operate. In particular, this will include both knowledge of specific perceptual cues and of the motor actions required for an optimum response to the situation presented by the cues (Anderson, 1990). The challenge for researchers investigating skilled performance is to produce an experimental paradigm for recording response measures while maintaining the contextual relevance that they need in order to access the performer's specific perceptual-motor knowledge for the skill of interest. This concern for ecological validity in experimental methodology is addressed here in the context of describing a computer-controlled interactive video simulation developed for applied sports research.

It is typically found that experts show more effective cognitive functioning when processing stimuli relevant for a particular skill than do novices (see, e.g., Abernethy, 1990; Houlston \& Lowes, 1993; Paull \& Glencross, 1997). Such experimental findings are highly task-specific and rest on the assumption that the relevant stimuli have been provided in the experimental procedure. If this fidelity (see Starkes \& Lindley, 1994) does not exist, the attenuation of ecological validity in the paradigm results in invalid data

The development of the technology and software and the production of video and other experimental resources for the research described in this paper was made possible by the support of the late Professor Denis Glencross, Head, School of Psychology, Curtin University of Technology. His enthusiasm for sport-related research in Australia will be missed. I.C. is Computer Systems Officer of the School of Psychology, Curtin University of Technology. R.G. is at the Department of Human Movement, University of Western Australia. Correspondence concerning this paper should be addressed to G. Paull, who is now at the Department of Exercise Science and Physical Education, Arizona State University, Tempe, AZ 85287-0404 (e-mail: gpaull@imap2.asu.edu). and possibly in misleading information about performance (Paull \& Fitzgerald, 1993).

The requirements, then, for an experimental paradigm examining perceptual factors in skilled behavior must include: (1) environments reproducing the perceptual information cues ${ }^{1}$ available in the usual performance situation; (2) dynamic displays of the action typical of the skill domain, including information about any alternative situations (uncertainty) that can arise; (3) realistic motorresponse actions that have, through practice, become coupled in a performer's memory to his/her perceptual system; (4) the ability to manipulate perceptual and probabilistic cues in the display with the expectancy that this will create changes in the subjects' responses; and (5) the recording of control, timing, and response data without interfering with subjects' execution of their usual actions for the skill.

Only after fulfilling these requirements can researchers be confident that any recorded data truly represent how a person behaves in the operative situations that usually occur. We suggest that interactive video is an effective method for meeting these standards for applied-skills research. The technological configuration for an initial system, developed at Curtin University of Technology, is presented here. The concept of the configuration is adaptable across a range of proprietary equipment, although control and software specifications will vary, both on account of the rapidly expanding variety of products and to accommodate different international standards and conventions. Therefore, the model is presented not as a "plug and play" configuration, but as a viable model for laboratory-based motor skills research that provides flexible application to various domains of skilled behavior. Selected results from two sports-performance studies are outlined below to illustrate the paradigm and to provide validity support. 


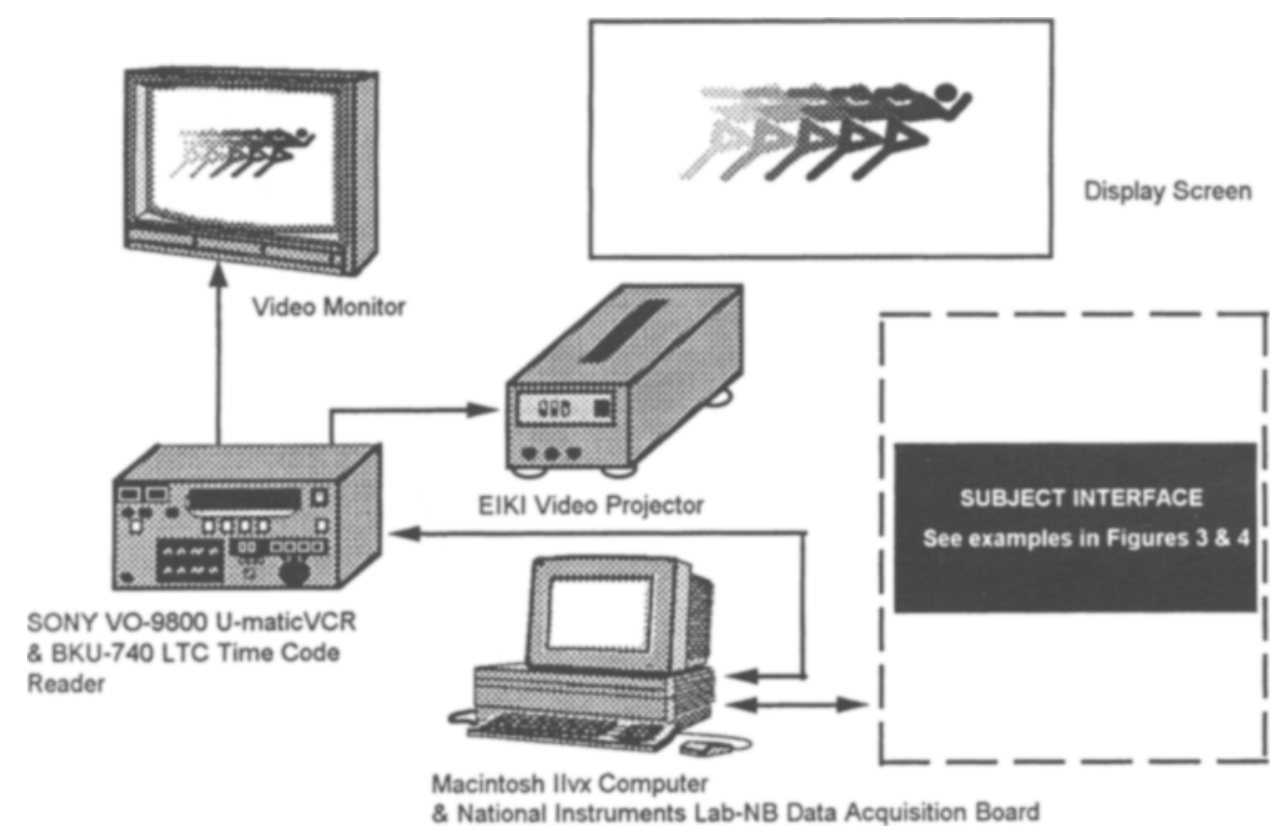

Figure 1. Pictorial layout of the interactive video configuration.

\section{AN ECOLOGICALLY VALID COMPUTER-CONTROLLED VIDEO SIMULATOR}

The basic tenet of an ecologically valid research paradigm is that subjects should execute the skilled performance with minimum interference from the system. To address this concern, the technological configuration shown in Figure 1 provided for centralized control of all equipment by one computer, thereby reducing experimenter intrusion into the testing environment. This computer coordinated the display and removal of video images and the acquisition of response signals, and it maintained time phasing via a communications bus linking all necessary components and subsystems, with minimum cabling visible or attached to subjects. Experimental displays were life-sized projections of action sequences provided from high-resolution video equipment. Data collection points for subjects' responses were embedded into a natural part of the sports apparatus or of the actions; data files that were generated during real-time performance were immediately stored to disk for later analysis.

The computer-controlled video simulator uses $\mathrm{Hi}$ band/SP U-matic format for playback and display. The U-matic (interlaced PAL at 25 frames/sec or 50 fields $/ \mathrm{sec}$ ) format provides a display resolution of 300 lines and includes a longitudinal time code (LTC) superimposed onto the videotape. This accommodates analysis of action sequences and the designation of "events" (visual cues) in experimental displays. Editing of the initial video produces displays of relevant action in blocks of time-coded trials for experimental playback.
The Sony U-matic VO-9800P VCR used for playback is equipped with a remote connection RS-422 serial port operating at $19.2 \mathrm{Kbits} / \mathrm{sec}$. This communications port provides access for an external computer to monitor and control all of the major VCR functions, such as cue forward, play, pause, cue backward, rewind, and stop (see Sony, 1990, for the full specification of this function). In addition, the ability to read time code information from the videotape via the serial port allows the controlling computer to synchronize video events with a master time clock. On any trial, therefore, response signals to the computer can be recorded as an elapsed time from the onset of the designated cue, along with any accuracy signal and other relevant performance data.

Each trial sequence in experimental displays begins with the videotape cued to the designated start-point time code. All start-point, end-point, and intertrial interval information is read from a data file containing the time codes designating these points. Figure 2 details a time line of events in each trial. When a VCR "PLAY" sequence from a cued position is started, the servo-mechanism controlling the playback video heads of the VCR is already energized, and the heads are in position. This means there is a minimum, and repeatable, time required for the tape drive mechanism to bring the tape to play speed, as was demonstrated in the 3,120 trials (practice plus experimental) from the sports studies described below. In those studies, measurement of the tape play command to tape play status duration $\left(t_{1}\right.$ to $\left.t_{3}\right)$, of the tape play command to read time code command duration $\left(t_{1}\right.$ to $\left.t_{5}\right)$, and of the difference in actual time code read at $t_{5}$ from the cued time code at $t_{0}$ were used to measure the repeatability of the trial 


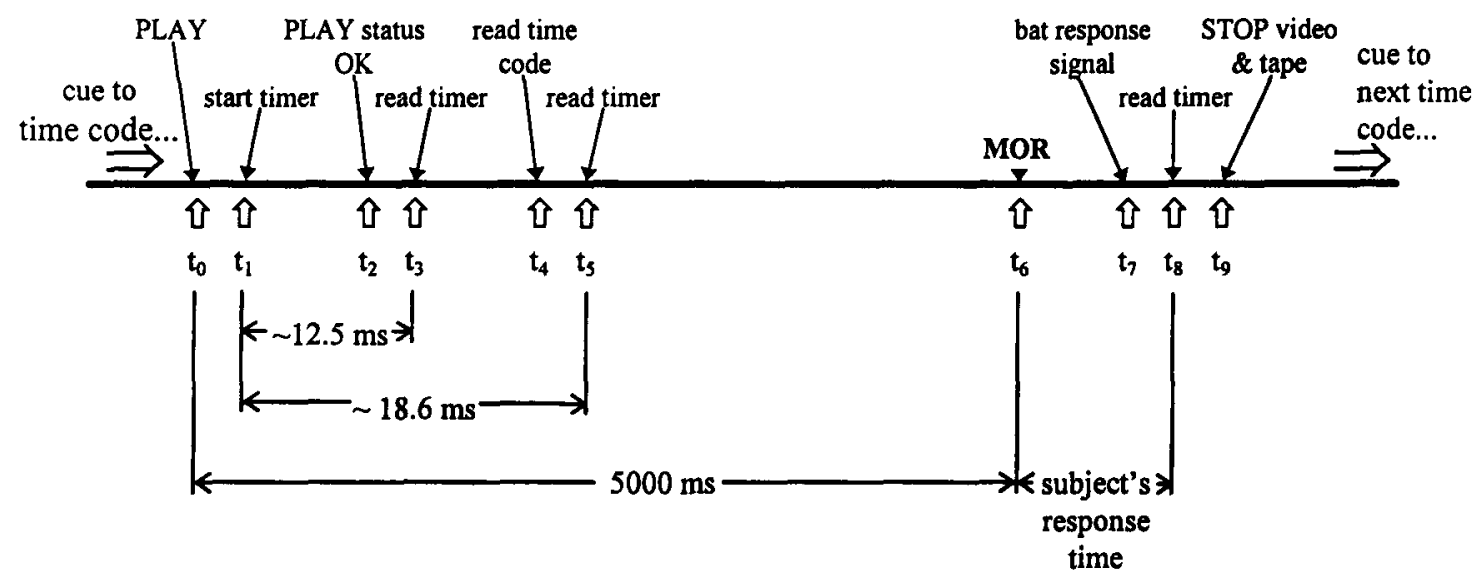

Figure 2. Time line of experimental equipment operations (not to scale).

process. Normal video accuracy was quoted by the manufacturer as \pm 1 frame (40 msec) (Sony, 1989); however, testing of the VCR and monitoring over the 2,760 experimental trials showed that the time from cue to tape play speed $\left(t_{1}\right.$ to $\left.t_{3}\right)$ was highly repeatable $(M=12.5 \mathrm{msec}, S D=$ $1.37 \mathrm{msec}$ ). Tape play command to time code read time ( $t_{1}$ to $t_{5}$ ) was nearly as repeatable, with a mean of $18.6 \mathrm{msec}$ and standard deviation of $2 \mathrm{msec}$. Both durations were very stable when compared with the specified absolute accuracy, implying a high degree of reliability of time measurement. The mean time code read command duration was $6.15 \mathrm{msec}$, with a standard deviation of $1.38 \mathrm{msec}$. Therefore, at least six time code reads could generally be accomplished using software polling of the video-frame LTC time code. This resolution of time code reading ensured that actual video events (perceptual cues) and external events (subjects' actions) were within milliseconds of recorded values. It was also found in all but a few cases (11 of 2,760 , or $0.003 \%$ ) that the first time code read frame value did equal the cue frame value; thus, it can safely be assumed that normal tape play speed was reached within the first two frames of playback and that highly reliable response measurements were made with a resolution in the order of milliseconds.

The EIKI video projector connects to the video out terminal of the VCR; it projects life-sized video images onto a suitable screen. This portable projector allows flexibility in the experimental layout, since it can be placed in any suitable location and provides a zoom range to adjust the projected display size. The initial image to be projected is formed on a three-layer color LCD screen in front of a single high-intensity lamp. The three layers of LCD represent each of the primary colors of blue, yellow, and red; a combination of these held "ON" provides the spectrum of colors required for the video images. For even less intrusion by equipment into the experimental environment, the projector has a "reverse image" mode allowing for backlit projection from behind the screen, if required.

Projection of the display as a life-sized image at a more natural distance from the subject is used to overcome some of the difficulties involved in presenting a three-dimensional event as a two-dimensional image. Evans (1970) reported that viewing the display at the actual distance of the filming (or videotaping, using current technology) preserves some of the ocular distance cues (e.g., binocular convergence of the eyes) which correspond to perspective constancies in the image, thereby further increasing the ecological validity of the paradigm.

Controlling the system is a Macintosh IIvx fitted with a National Instruments Lab-NB data-acquisition board. The Lab-NB board has 24 TTL-compatible digital ports, a 12-bit analog-to-digital converter with 8 analog ports, and six 16-bit counter/timer channels. This card provides the hardware interface to transducers in the recording apparatus signaling subjects' response actions. The software program provides experimenters with a menu-driven standard Macintosh interface. It was developed with Symantec Think Version C 5.0; it uses proprietary library routines for the operation of the Lab-NB and specially written software libraries in C or LabView (see National Instruments, 1991) for the operation of the Sony VCR. After the program is started, an Option menu is provided for selecting which segments of the videotape are to be used as trials (accommodating randomization and counterbalancing requirements) and for loading relevant time code information from a file matching the tape in the VCR. For each tape segment, time code information includes a start time, an end time, and a cue marker (e.g., in sports research, the frame that shows the moment of ball release from a pitcher's hand-MOR). Trials begin with a selection from the Run menu, after which the operation of the experimental and data recording apparatus is automatic. For each trial, the videotape tracks to the segment indicated by the start-time code, the subject is alerted with a tone of trial commencement, and the VCR is then set to Play until the end-time code is reached or until a response signal causes a Stop condition to be established. During this sequence, the response time of the subject is measured as the difference between the cue marker time code and the response signal detected on the transducers of the ex- 


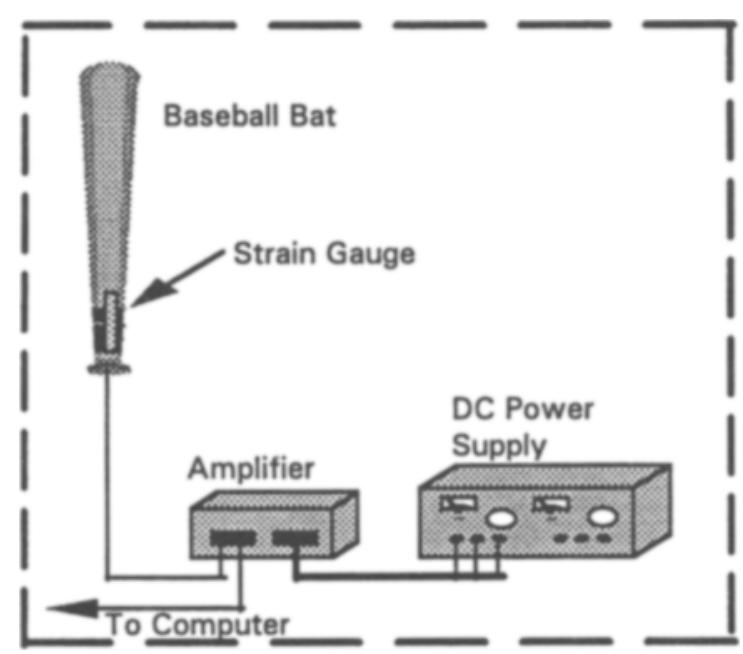

Figure 3. Baseball batting data signaling apparatus.

perimental apparatus. The response-time information is then stored to file.

Control programs for the two studies described below were written specifically for the proprietary equipment used in the procedures. Details of the codes used are available from the second author.

The transducers used as the response apparatus for the two studies presented in this paper are shown in Figures 3 and 4. Operation of each configuration is described in the appropriate experimental procedures below.

To illustrate the control system and hardware interfacing of components, two studies of skilled performance in sport are described below. Baseball is a sport suited to this paradigm because the display available in the laboratory setting is most appropriate to "closed" skills, in which an opponent or the cues of interest are at a relatively fixed direction and distance.

\section{STUDY 1 \\ Baseball Batting}

The objective of this study was to identify specific visual information used by batters to make decisions about a pitched ball. Batters were also tested for their ability to set probabilities about what type of pitch would be thrown next (see Paull \& Glencross, 1997, for a full explanation and for the results of this study). There were two experiments in the study, using different video displays and procedures to compare the performance of a group of expert batters with that of baseball novices.

Experiment 1 examined the decision times and the accuracy of batters viewing pitches on a video monitor (fullsized video projection was introduced later; see Study 2 for details). Further, their ability to set probabilities about the next pitch was tested by providing strategic game information for half of the trials. Expected outcomes from Experiment 1 were (1) that expert batters would have faster decision times and would be more accurate than novices were, and (2) that, when game strategic informa- tion was presented, experts would use this information to set generally accurate probabilities about forthcoming action and thus would improve their decision-time and accuracy scores, whereas novices would not.

The second experiment used video that was manipulated to determine the value, throughout the sequence of a pitch, of visual information from the pitcher in his action. The display consisted of video edited to provide an occlusion of information whereby, at five designated points in the pitch delivery, the pitcher and ball disappeared, and only blank stadium background remained. Each display was occluded at a different point in time - that is, at $T_{1}$, two frames $(-80 \mathrm{msec})$ before MOR of the ball from the pitcher's hand; at $T_{2}$, at MOR; at $T_{3}$, two frames $(+80 \mathrm{msec})$ after MOR; at $T_{4}$, four frames ( $\left.+160 \mathrm{msec}\right)$ after MOR; or at $T_{5}$, six frames $(+240 \mathrm{msec})$ after MOR.

It was expected that expert batters would use visual cues provided in advance of MOR and from early in the pitch delivery. Since novices were not expected to use this early information, it was hypothesized that experts would have greater accuracy than novices would when occlusion occurred earlier in the pitch delivery.

For video recording, a pitcher from the Australian Baseball League was used. The pitcher was videotaped at a shutter speed of 1/500th of a second (to provide crisp, distortion-free images), from the aspect of a right-handed batter. In the laboratory, the video sequences were displayed to the batter on a high-resolution 64-cm monitor. Subjects were positioned in a batting stance at a distance from the monitor such that the pitcher's image subtended an angle to the eye of $6^{\circ} 17^{\prime}$, which is the approximate size for an 180-cm-tall pitcher at a distance to the pitcher's plate of $18.44 \mathrm{~m}$. The video monitor was elevated to a height that produced a view equivalent to that of a pitcher positioned on a standard $35-\mathrm{cm}$-high mound. Subjects could step and swing the bat, if this helped to provide a more natural approach to the trials.

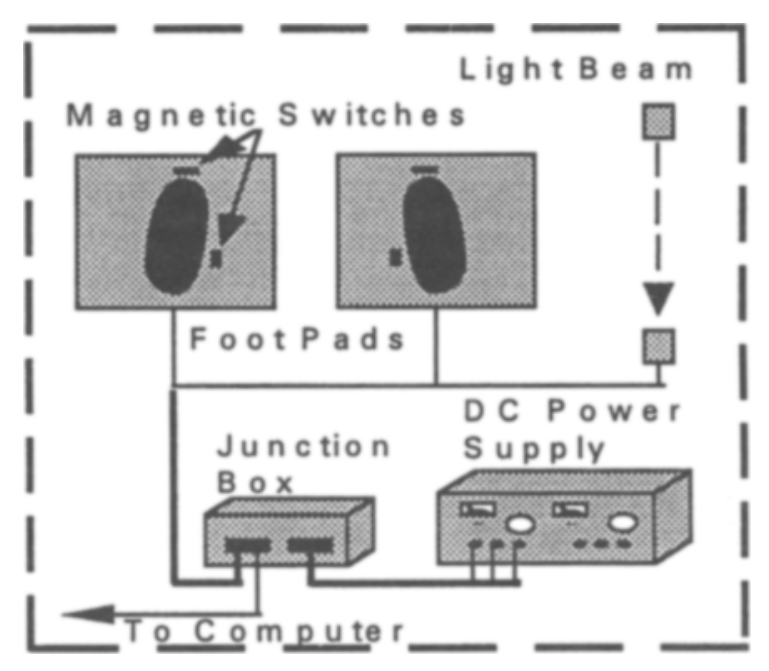

Figure 4. Basestealing data signaling apparatus. 


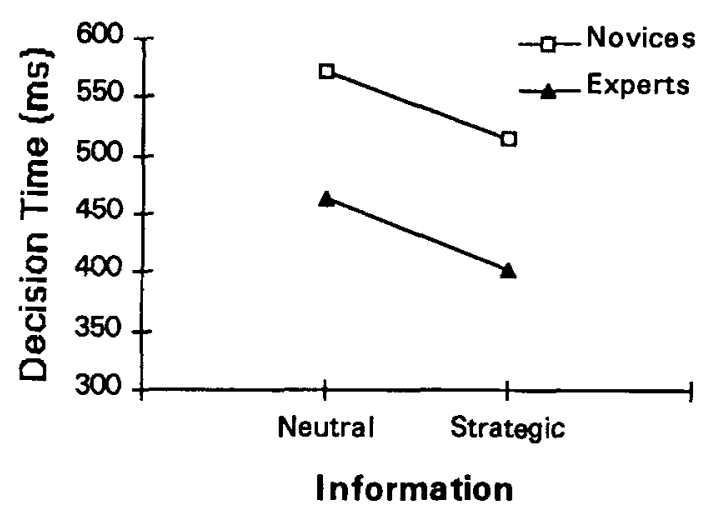

Figure 5. Decision times for expert and novice batters. From "Expert Perception and Decision Making in Baseball," by G. C. Paull and D. J. Glencross, 1997, International Journal of Sport Psychology, 28, p. 45. Copyright 1997 by Edizioni Luigi Pozzi. Reprinted with permission.

The earliest consistent physical response representing a decision by a batter to swing at a pitch was a tightening of his grip on the bat. Therefore, for the decision-time response (DT), the baseball bat shown in Figure 3 was fitted with a strain gauge. A change in grip pressure by the batter was sensed on the strain gauge connected as a quarter bridge and was amplified by a stable balanced amplifier with gain of $A_{v}=1,000$. This signal was then fed to the analog input of a 12-bit analog-to-digital converter in the data acquisition card, generally using a sampling rate of $1.0 \mathrm{msec}$ (adjustable as a software parameter). This signal was in the range of $0-100 \mathrm{mV}$, depending on grip pressure, with DT registered when a preset threshold was reached. Given the different resting grip pressures of individual batters, the threshold could be calibrated before trials were run.

In the experimental situation, receipt of a response signal from the strain gauge in the handle of the bat caused the video signal to the monitor to be broken (VCR "Stop"), thus precluding any further information on ball flight. After the monitor blanked, subjects provided a number from a grid of the strike zone that represented their prediction of what the ball's position would be as it passed home plate. On probability-setting strategic pitches, the subject was shown a game card depicting the state of a hypothetical game before each trial. Therefore, scoring for each subject comprised DT in milliseconds (automatically recorded during each trial) and predictions of ball location (calculated to an "error" score).

In the occlusion study (Experiment 2), no decision time was recorded, as the stimulus (information from the pitcher's actions and the ball flight) was removed from the display at a predetermined time in the pitch, as noted for $T_{1}$ to $T_{5}$ above.

Thirty right-handed subjects participated in the study, 15 in the expert group (players with a batting average $>.300$ for the previous season) and 15 novices (players who had never played higher than B grade, the fourth level in their competition).

Figures 5 and 6 summarize the results of the first experiment. As expected, it was found that experts were significantly faster in their decision times [expert $M=$ $463 \mathrm{msec}$, novice $M=571 \mathrm{msec} ; F(1,28)=10.45, p<.01]$. This superior performance did not occur at the expense of accuracy; experts were significantly more accurate than the novices $[F(1,28)=9.96, p<.01]$. It was also found that strategic information facilitated faster and more accurate decision making $\left[F_{\mathrm{DT}}(1,28)=55.37, p<.001 ; F_{\text {error }}\right.$ $(1,28)=7.90, p<.01]$.

The overall results across the stages of information occlusion from Experiment 2 are given in Figure 7. It was hypothesized that experts would be able to use information from earlier in the pitch than novices would. As shown, experts were generally more accurate than novices were after the moment of delivery of the pitch (although the only significant change is for experts between $T_{4}$ and $T_{5}[F(1,14)=7.16, p<.05]$. Improvement in accuracy scores by both groups was not shown until after $T_{3}$-that is, after $80 \mathrm{msec}$ (or about $3 \mathrm{~m}$ ) of ball flight. From $T_{3}$, the diverging trends in scores indicated that experts had a somewhat greater ability to use early ball flight cues in determining the trajectory of the ball; these differences between groups are significant at $T_{4}$ and $T_{5}\left[F_{T_{4}}(1,28)=\right.$ $\left.6.25, p<.05 ; F_{T_{5}}(1,28)=8.28, p<.01\right]$.

\section{STUDY 2}

\section{Baseball Basestealing}

The second study examined the relative value of different visual cue sources a baserunner might use in deciding to either steal or return to a base. The value of cues depends on how much information they provide about the pitcher's intention to either pitch to the batter or throw to the base the runner occupies. There has been considerable debate in the coaching literature about the relevance of various cues (Grove, 1989), and the study addressed this issue.

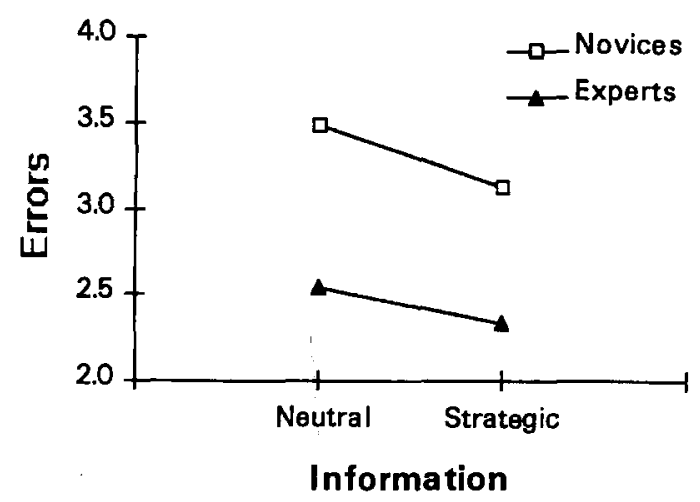

Figure 6. Error scores for expert and novice batters. From "Expert Perception and Decision Making in Baseball," by G. C. Paull and D. J. Glencross, 1997, International Journal of Sport Psychology, 28, p. 45. Copyright 1997 by Edizioni Luigi Pozzi. Reprinted with permission. 


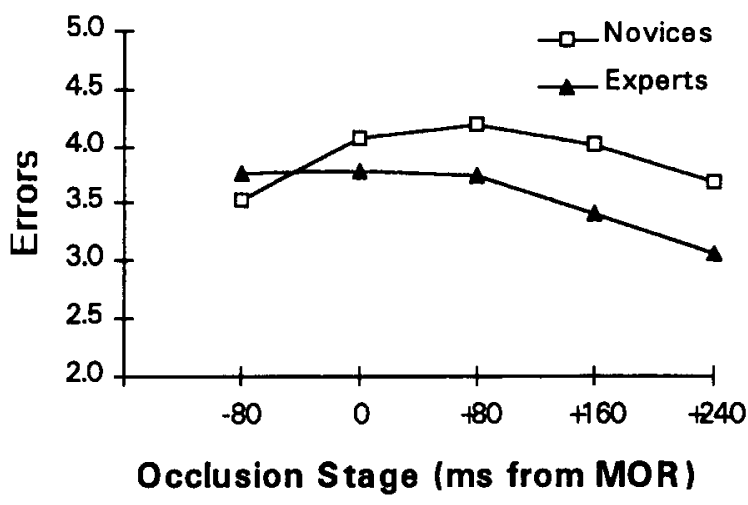

Figure 7. Error scores across stages of occlusion. From "Expert Perception and Decision Making in Baseball," by G. C. Paull and D. J. Glencross, 1997, International Journal of Sport Psychology, 28, p. 48. Copyright 1997 by Edizioni Luigi Pozzi. Reprinted with permission.

This experiment was concerned, then, with the response times of runners to cues from different regions of the pitcher's body and with whether the value of specific cues varied between right- and left-handed pitchers. The video display was edited into two sets (right-handed pitchers and left-handed pitchers) of five arrays to direct attention to various segments $\left(S_{1}-S_{5}\right)$ of the display. Segmenting of the display was achieved with a panel superimposed on the screen to mask half of the pitcher's body at a time. The panel was a neutral olive green color so that it would blend in with the stadium background for the area behind the pitcher. Therefore, subjects would be shown views of the top, bottom, front half (down the trouser seam), and back half of the pitcher throughout the series of trials. The occlusion of cues was maintained during the critical period prior to the pitcher's committing to an action, and movement out of the mask (for front/back masking) occurred with the natural action of pitching or picking-off.

For the basestealing study, several pitchers from the Australian Baseball League were videotaped, at a shutter speed of $1 / 500 \mathrm{sec}$, from a baserunner's position when leading off first base (i.e., approximately $2.5 \mathrm{~m}$ from the base). In the laboratory, the video was projected onto a screen $7 \mathrm{~m}$ from the subject, in a direction equivalent to the position of a pitcher on the mound of a baseball infield. As in the previous study, the pitcher's image was the same size (approximately $6^{\circ} 17^{\prime}$ on the eye) as in the live game situation.

The footpads shown in Figure 4 were each fitted with two magnetic reed proximity switches, one near the ball of the foot and one at the heel, to signal the subjects' response times. The reed switches were closed when mag- nets attached to the subjects' feet were in close proximity and released when the subjects moved from the pads in response to the video action. The four switch values were fed through the junction box to digital ports in the dataacquisition card. The light beam optical switch was positioned on the path of the runner when returning to the base (i.e., on a pick-off trial). Accuracy of the direction of movement on any trial was determined (1) from the registration in software of whether a trial was a pitch or a pickoff and (2) from either a signal from the optical switch (= Error [subject should have run toward second base]) or no signal in the time-out period $(2 \mathrm{sec})(=$ Correct) on a pitch, or either a signal from the optical switch (= Correct) or no signal in $2 \mathrm{sec}$ (= Error) on a pick-off. The output of this switch was latched in the junction box before being directed to a digital port in the data-acquisition card.

Sixteen baseball players from the West Australian Baseball League (WABL) participated in the study. A comparison of these subjects' time to respond (RT) across the five displays of pitchers is given in Table 1. Apart from any interest in the various segments of the display [which produced differences among the times recorded; $F(4,12)=$ $10.12, p<.01]$, the ecological validity of the procedures was supported by the difference in response times found for right- and left-handed pitchers $[F(1,15)=27.45, p<$ $.001]$. As would be expected in a game situation, the advantage enjoyed by a left-handed pitcher in facing first base precludes the runner's attempting to steal until later in the pitcher's action.

\section{FUTURE DEVELOPMENTS FOR TECHNOLOGY-BASED APPLIED SPORTS RESEARCH}

The video-based simulation used to investigate baseball skills overcame many of the ecological-validity difficulties previously seen in applied sports research. The present configuration, in particular, demonstrated a cost-effective use of technology where more elaborate systems were not available. It is expected that higher level systems will come within the economic reach of smaller research centers in time; the following factors should be considered for future applications of the technology.

First, digital video offers great potential for the simulation of particular environments. The media used to store digital video include video disk, CD-ROM, or computer hard disk, which provide fast, nonlinear access to any location on the media. With this feature, the start and end points of sequences to be presented as stimuli are easily determined and accessed (in random order, if necessary), which reduces the need to edit unwanted scenes, which

Table 1

Average Response Times (in Milliseconds)

Across Different Views of the Pitcher

\begin{tabular}{|c|c|c|c|c|c|}
\hline Pitcher & $\begin{array}{c}\text { Full } \\
\text { View }\end{array}$ & $\begin{array}{l}\text { Top } \\
\text { Only }\end{array}$ & $\begin{array}{c}\text { Bottom } \\
\text { Only }\end{array}$ & $\begin{array}{c}\text { Front } \\
\text { Half Only }\end{array}$ & $\begin{array}{c}\text { Back } \\
\text { Half Only }\end{array}$ \\
\hline Left-handed & 776.5 & 709.9 & 783.4 & 795.3 & 716.7 \\
\hline Right-handed & 424.2 & 430.3 & 426.6 & 485.1 & 514.4 \\
\hline
\end{tabular}


are left isolated outside the designated sequences. Digital video also provides opportunities for computerized editing and dubbing to extend or augment the sensory information provided to subjects. Incorporating graphics, sound, and text (see the following paragraph for specific examples of this) synchronized to the video sequences increases the number of experimental possibilities within a paradigm. Nonlinear access also eliminates the transit time that occurs in standard videocassettes when cuing to the start points of sequences. Further, access is virtually silent, which eliminates tape pick-up, motor winding, and reversal noise from a VCR, all of which intrude on the contextual reality of the experimental environment. The playback medium for digital video is incorporated directly into the computer through the use of software such as Apple's QuickTime, now developed to provide real-time playback in suitably equipped computers. This caveat regarding sufficient processing power and computer memory is less necessary with the availability of modern multimedia computers, but the problem must be addressed to ensure the uninterrupted information flow to subjects and, thus, the maintenance of ecological validity for the usual performance environment.

Another enhancement, further reducing experimenter intrusion, can be provided by including instructions and nonvisual information in the videotape recording. For the strategic game information in Study 1, this could have been presented as a pictorial view of the field showing any baserunners and a scoreboard with relevant game-state information. Other information may also be included on tape, providing, for example, crowd noises, engine pitch for pilot and driver testing, and many other sources of sensory information that have an impact on performance in motor-skills environments.

The use of full-size images at actual distances is necessary to the paradigm. It has been explained above (see Evans, 1970) that perceptual cues from eye kinesthesis are then consistent with features of the display. Further, reallife spatial relationships are maintained in full-sized displays. For example, in facing live baseball pitches, the trajectory of a curve ball may be relatively high, and then "break" to pass about calf height of the batter. On a small screen, this motion is depicted, but, after breaking, the ball is still somewhere around waist high on a screen elevated to chest height; this does not provide the "feel" of the actual batting situation.

To reduce the need for verbal responses by subjects, a three-dimensional motion analysis system would provide more accurate response measures. First, this system could have tracked the bat head in Study 1 (Experiment 1) as the batter "hit" the pitch. The position of the bat head in space might have been a more precise measure of accuracy than was the grid error score derived from the verbal reports of subjects. This is important, given the lack of consistent support for elite performers to reliably translate what is usually a skeletomuscular response into a verbal description of their actions (Berry \& Broadbent, 1984; Nisbett \& DeCamp Wilson, 1977).
Second, a motion analysis apparatus would be capable of monitoring many body parts of the subjects in Study 2, thereby recording the first movement of sufficient magnitude to indicate the "decision" response. It is quite likely that the foot switches used in that study in fact lag behind other body movements (perhaps of the knees, of the shoulders, or even facial expressions) in indicating DT. Motion analysis devices would allow many simultaneous recordings to detect a subject's first movement, no matter where it originated.

Finally, interactive video simulation has an emerging role in training programs. A major determinant of skilled performance is the amount of consistent, deliberate practice undertaken (Ericsson, Krampe, \& Tesch-Römer, 1993; Schneider \& Fisk, 1982). In many bat and ball sports, the inability to provide such consistent visual information for a whole team in one training session can be a major handicap in the development of elite skills. On this point, Starkes and Lindley (1994) have reviewed learning via video simulation. Their study supported video training of athletes, concluding that, in basketball, there were positive effects for players who received off-court video instruction in game skills. Further support comes from Hubbard (1994), who trained bobsled drivers in a video/electromechanical configuration that simulated various international tracks. In an extension of the paradigm, Seymour, Stahl, Levine, Ingram, and Smith (1994) have investigated various video-based simulators used in firearm training with respect to their possible use in basic research into law-enforcement skills.

Using the technology described in this paper for a learning station would maximize interaction between the user and the stimulus display. In a sports example, a baseball player would set the computer to present a random array of pitch types taken from a library of displays on video disc. After a number of pitches, the batter may ask for a selection of the displays at which he or she has performed poorly in the first bracket (e.g., left-handed pitchers, curveballs, etc.). With the ability to train in any weather and at any time without the aid of expert pitchers, it may be expected that a developing batter would perform enough repetitions to accelerate the acquisition of batting skill above what could be accomplished from only live batting practice.

\section{CONCLUSION}

This paper has outlined the development of a costeffective interactive video simulator for applied motor skills research. At this stage, the computer, video, and interfacing technology is able to provide contextually relevant displays of perceptual "stimuli" in a stable control, timing, and communication configuration. Beginning with this technological platform, various subject-interface mechanisms can be designed to transmit response data to the computer from the natural actions of subjects while they respond to the video display.

The studies described illustrate the success to date in achieving ecological validity for examining baseball skills. In both batting and basestealing, the procedures and 
operations for researchers and subjects suggest high levels of fidelity - that is, the environment was a true representation of the actual skill environment - in the laboratorybased simulation. Moreover, the recording of variations in perceptual-motor operation due to the expertise differences of subjects indicates the simulator's potential for examining many types of behavior throughout the stages of skill acquisition. Certainly, the simulator can make a contribution to understanding expert performance and, in skill development programs, to accelerating learning of the essential information that facilitates the highest levels of performance.

\section{REFERENCES}

ABERNETHY, B. (1990). Anticipation in squash: Differences in advance cue utilisation between expert and novice players. Journal of Sports Sciences, 8, 17-34.

ANDERSON, J. R. (1990). Cognitive psychology and its implications (3rd ed.). New York: W. H. Freeman.

BERRY, D. C., \& BROADBENT, D. E. (1984). On the relationship between task performance and associated verbalisable knowledge. Quarterly Journal of Experimental Psychology, 36A, 209-231.

Ericsson, K. A., Krampe, R. T., \& Tesch-Römer, C. (1993). The role of deliberate practice in the acquisition of expert performance. Psychological Review, 100, 363-406.

Evans, L. (1970). Automobile-speed estimation using movie-film simulation. Ergonomics, 13, 231-237.

GRove, J. R. (1989). Visual processes in baseball: A glance at the literature and a comment on research possibilities. Journal of Applied Research in Coaching \& Athletics, 4, 176-194.

Houlston, D. R., \& LOWEs, R. (1993). Anticipatory cue utilisation processes amongst expert and non-expert wicketkeepers in cricket. International Journal of Sport Psychology, 24, 59-73.

Hubbard, M. (1994). Simulating sensitive dynamic control of a bobsled. In N. De Mestre (Ed.), Second Conference on Mathematics and
Computers in Sport (pp. 1-6). Gold Coast, QLD, Australia: Bond University, School of Information Technology.

National InStruments (1991). LabView 2 user manual. Austin, TX: Author.

Nisbett, R. E., \& DeCamp Wilson, T. (1977). Telling more than we can know: Verbal reports on mental processes. Psychological Review, 84, 231-259.

Paull, G. C., \& Fitzgerald, D. (1993). Addressing ecological validity through inter-active video based sport simulation. In S. Serpa (Ed.), Proceedings of the 8th World Congress of Sports Psychology (pp. 175-179). Lisbon: ISSP.

Paull, G. C., \& Glencross, D. J. (1997). Expert perception and decision making in baseball. International Journal of Sport Psychology, 28, 35-56.

SCHNEIDER, W., \& FISK, A. D. (1982). Degree of consistent training: Improvements in search performance and automatic process development. Perception \& Psychophysics, 31, 160-168.

Seymour, G. O., Stahl, J. M., Levine, S. L., Ingram, J. L., \& Smith, R. F. (1994). Modifying law enforcement training simulators for use in basic research. Behavior Research Methods, Instruments, \& Computers, 26, 266-268.

SONY (1989). Technical manual for VO-9800P VCR. Japan: Author. SONY (1990). Video recorder VVH-2XXX series: Protocol of remote- 2 (9 pin) connector (rev. ed.). Japan: Author.

StARKES, J. L., \& LiNDLEY, S. (1994). Can we hasten expertise by video simulations? Quest, 46, 211-222.

\section{NOTE}

1. Generally, visual cues are described in this paper and throughout the supporting research cited herein. There is a need to extend such investigation to other perceptual modalities (especially those used by athletes in complex sporting environments) that influence motor performance. Suggestions for suitable procedures to address this need are discussed later in the paper.

(Manuscript received April 24, 1995; revision accepted for publication December 11, 1996.) 